\section{Microbiological contamination in three large-scale pig slaughterhouses in Northern Italy}

\author{
Pierluigi Di Ciccio, ${ }^{1}$ \\ Maria Cristina Ossiprandi, ${ }^{2}$ \\ Emanuela Zanardi, ${ }^{\text {Sergio Ghidini, }}$ \\ Giancarlo Belluzzi, ${ }^{3}$ Alberto Vergara, ${ }^{4}$ \\ Adriana lanieri ${ }^{1}$ \\ 'Department of Food Science, University \\ of Parma, Parma; 'Department of \\ Veterinary Science, University of Parma; \\ ${ }^{3}$ Ministry of Health, Parma; ${ }^{4}$ Faculty of \\ Veterinary Medicine, University of \\ Teramo, Teramo, Italy
}

\section{Abstract}

The aim of this survey was to obtain data on microbiological contamination of pig carcasses and environments in three large-scale Italian slaughterhouses (identified as A-B-C) located in Northern Italy. Each slaughterhouse was visited six times. Five carcasses and three different sites of the slaughterhouse (before and during slaughter) were sampled on each sampling day. A single pooled caecal sample was taken on each sampling day. A total of 90 carcasses, 108 environmental samples and 18 caecal samples were collected. Samples from pig carcasses and slaughterhouse environment were analyzed for total viable count (TVC), Enterobacteriaceae count (EBC) and Salmonella. The caecal contents were examined for Salmonella. Carcasses from slaughterhouse A presented the greatest TVC and EBC mean $\log$ value, whereas environmental samples collected during slaughter activities from slaughterhouse $\mathrm{C}$ showed the greatest TVC and $\mathrm{EBC}$ mean $\log$ value. As far as the environmental samples collected before slaughter activities are concerned, an average up to $6 \log _{10}$ colony forming unit (CFU) $/ \mathrm{cm}^{2}$ TVC in two slaughter plants (A and C) and $5 \log _{10} \mathrm{CFU} / \mathrm{cm}^{2}$ TVC in one slaughter plant (B) was detected. Salmonella was recovered in two slaughterhouses (A and B). Four different Salmonella serotypes were detected in the positive samples (11). Within serotype $S$. Rissen and $S$. Typhimurium monophasic-variant isolates, two pulsed-field gel electrophoresis patterns were identified. The findings in this survey suggest that carcass contamination is influenced by the slaughterhouse plant and this could be a result of differences in line speed. The results of environmental sampling have not shown an association with the slaughterhouse plant.

\section{Introduction}

Pork is the most frequently consumed meat in the European Union (EU) and its consumption has increased in Mediterranean countries (Valero et al., 2014). Italian pig breeders are specialized in the heavy pig: they must weigh around $150 \mathrm{~kg}$ and greater than 9 months of age, which represents $90 \%$ of the Italian pig population. Heavy pig farming is mainly located in Northern Italy. In addition, this area includes a territory with the largest production of production denomination of origin Parma ham. Since 2005, the European Union (EU) has established strict microbiological criteria for pig carcasses (Reg. n. 2073/2005; European Commission, 2005). The Regulation specifies process hygiene criteria for total viable counts (TVC), Enterobacteriaceae count (EBC) and Salmonella for the post-evisceration and prechilling stages, which provide useful data for the validation and verification of HACCP procedures and other hygiene control measures employed in the pork industry. In order to reduce the Salmonella prevalence on pig carcasses, the interpretative criteria on hygiene during slaughter were changed in accordance with the Regulation (EU) n.217/2014 (European Commission, 2014). In particular, the number of samples (the $c$ value) where the presence of salmonella is detected, was reduced. Hygiene standards may vary between slaughterhouses in relation to slaughtering practices and slaughterhouse equipment (Wilhelm et al., 2011). It is well known that Salmonella is one of the main biological hazards in pork products (EFSA, 2012). Salmonella can enter the supply chain in many stages of the process, e.g. in the primary stages by feed, people or rodents, during transportation by infected trucks or in the slaughterhouse by cross-contamination of infected animals. Pigs generally carry Salmonella asymptomatically (Duggan et al., 2010). The slaughtering stage is considered to have the greatest impact on the number of contaminated carcasses as shown by several authors. Salmonella prevalence in slaughter pigs has been investigated in a number of studies. In the EU, $10.3 \%$ of the slaughter pigs were found to carry Salmonella in the lymphnodes and $8.3 \%$ of the carcasses were contaminated with Salmonella (EFSA, 2008a, 2008b, 2009). In pigs, the most common serotypes are $S$. Typhimurium and $S$. Derby (Gomes-Neves et al., 2012). Currently, there is a paucity of information about the microbiological contamination of carcasses and environment in large scale Italian pig slaughterhouses with high processing speed (up to 450 pigs/hour). With regard to this, the aim of this survey was to obtain data on microbiological contamination of pig carcasses and slaughterhouse environ-
Correspondence: Pierluigi Di Ciccio, Department of Food Science, University of Parma, via del Taglio 10, 43125, Parma, Italy.

Tel: +39.0521.032753 - Fax: +39.0521.032752.

E-mail: pierluigialdo.diciccio@nemo.unipr.it

Key words: Pig slaughterhouse; Pig carcass; Total viable count; Enterobacteriaceae; Salmonella.

Acknowledgements: the authors would like to thank Dr.ssa Cinzia Reverberi from the Department of Veterinary Science, University of Parma (Italy) and Dr. Stefano Pongolini from Institute for Experimental Veterinary Medicine of Lombardy and Emilia Romagna, Parma (Italy) for their technical assistance.

Received for publication: 2 March 2016.

Revision received: 30 May 2016.

Accepted for publication: 30 May 2016.

This work is licensed under a Creative Commons Attribution-NonCommercial 4.0 International License (CC BY-NC 4.0).

CC Copyright P. Di Ciccio et al., 2016

Licensee PAGEPress, Italy

Italian Journal of Food Safety 2016; 5:6151

doi:10.4081/ijfs.2016.6151

ment in three large-scale Italian slaughterhouses (identified as A-B-C) located in Northern Italy.

\section{Materials and Methods}

\section{Study design and sample collection}

The investigated area includes a territory with the largest production of PDO Parma ham, i.e. the so-called Food Valley (extending in Piedmont, Lombardy, Emilia-Romagna and Veneto regions), where more than $90 \%$ of slaughterhouses slaughter pigs for Parma ham production. In 2014, 7,963,134 heavy pigs for PDO Parma ham production were slaughtered in Italy. About $93 \%$ of these pigs were slaughtered in 18 large-scale pig slaughterhouses located in Northern Italy (http://www.prosciuttodiparma.com/pdf/en_UK/Specifications.pdf). In this survey, three pig slaughterhouses (identified as A-B-C) with a capacity of 550,000 heavy pigs per year were randomly selected. The three slaughterhouses were comparable from the point of view of the slaughter practices. The number of pigs slaughtered per hour was 350 in two slaughterhouses (B and C) and 450 in the slaughterhouse A. Each slaughterhouse was visited six times. Five carcasses and three environmental sites (before and during slaughter) were sampled on each sampling day. Additionally, a single pooled sample (caecal samples from pigs in a single batch) was taken on each sampling day. A total of 90 carcasses, 
108 environmental samples and 18 caecal samples were collected.

\section{Carcasses}

A total of 30 pig carcasses ( 5 carcasses on each sampling day) were sampled at each slaughterhouse. The carcasses were randomly selected, taking at least a 10 animals interval between one another. All the slaughtered pigs were certified to be processed for typical PDO ham production. They were identified by date, farm code and area of origin, and followed along the slaughtering line for sampling after dressing but before chilling. Samples were obtained by pre-hydrated sponges (International PBI S.P.A., Milan, Italy) with Buffered Peptone Water (BPW; Oxoid, Milan, Italy) $(10 \mathrm{~mL})$ from four different sites $(4 \times 100$ $\mathrm{cm}^{2}$ ): hind limb, abdomen lateral (belly), middorsal region (mid-back) and jowl. All samples (two sponges for each carcass) were sent to the laboratory in cooled containers within the same day for their analysis.

\section{Caecal contents}

A total of six pooled caecal samples (225 g) were taken at each slaughterhouse after evisceration. On each sampling day, caecal samples were collected aseptically from different pigs of the same batch whose carcasses were sampled. Finally, all samples were individually packed, registered and kept at a temperature $+4^{\circ} \mathrm{C}$ during storage and transportation to the laboratory.

\section{Environmental samples}

A total of 36 environmental samples (18 before slaughter activities and 18 during slaughter activities) were collected at each slaughterhouse. Three different points of the slaughter line (floor after bleeding, gut container and run off pit/drain well) were evaluated in two sampling rounds. The first sampling round was done early in the morning before the processing beginning; the second sampling round was done during slaughter activities. Briefly, a $100 \mathrm{~cm}^{2}$ surface per site using a template was sampled using sponges pre-soaked in $10 \mathrm{~mL}$ of BPW (Oxoid) by collecting surface swabs in floor after the bleeding stage, in runoff pit and in gut container. All samples were stored at $+4^{\circ} \mathrm{C}$ and returned to the laboratory within the same day for their analysis.

\section{Enumeration of microorganisms: total viable count and \\ Enterobacteriaceae count}

In the laboratory, each sample from environment and the pooled sponges (four) of each carcass was homogenized for $120 \mathrm{~s}$ in BPW (90 $\mathrm{mL}$ ) in a stomacher machine (Interscience, Paris, France). All suspensions were diluted and plated on plate count agar (PCA; Oxoid) and violet red bile glucose agar (VRBGA; Oxoid) for enumeration of the TVC (UNI EN ISO 4833) and EBC (ISO 21528-2). The PCA and VRBGA plates were incubated for $72 \mathrm{~h}$ at $30^{\circ} \mathrm{C}$ and for $48 \mathrm{~h}$ at $37^{\circ} \mathrm{C}$, respectively. The TVC and EBC counts for each sample were expressed as $\log _{10}$ colony forming unit (CFU) $/ \mathrm{cm}^{2}$.

\section{Salmonella isolation, identification and serotyping}

Slaughterhouse and carcass samples were then pre-enriched in BPW. The sponges resulting from carcass and environment swabs in BPW $(90 \mathrm{~mL})$ were incubated at $37^{\circ} \mathrm{C}$ for $24 \mathrm{~h}$, whereas the pool of fecal samples ( $25 \mathrm{~g}$ ) were homogenized for $120 \mathrm{~s}$ in BPW (225 mL) in a stomacher machine (Interscience) and incubated at $37^{\circ} \mathrm{C}$ for $24 \mathrm{~h}$. After the incubation period, all samples were analyzed according to the Salmonella Precis method (Oxoid) validated by AFNOR. Presumptive colonies were examined by Gram-stain and submitted to biochemical identification with the API ID-32E system (bioMérieux, Marcy l'Etoile, France). All isolates were sent to the laboratories of the Istituto Zooprofilattico Sperimentale della Lombardia e dell'Emilia-Romagna in Brescia (Italy), serotyped by agglutination tests with specific $\mathrm{O}$ and $\mathrm{H}$ antisera (Edwards, 1962; Morris et al., 1972; Spicer, 1956) and classified according to the Kauffmann-White scheme (Grimont and Weill, 2007). Finally, Genomic DNA was analyzed by pulsed-field gel electrophoresis (PFGE), using the following restriction enzymes: XbaI (50U/sample) BlnI/AvrII (30U/sample), according to the PULSENET protocol (PULSENET, 2010).

\section{Statistical analysis}

The resultant counts for TVC and EBC were expressed as $\log _{10} \mathrm{CFU} / \mathrm{cm}^{2}$. Microbiological counts data among slaughterhouses were compared. In particular, the data were analyzed using IBM SPSS (Armonk, NY, USA) statistical software. The analysis of variance according to Sheffè post-hoc test was performed. Mean differences were considered significant when $\mathrm{P}<0.05$ (Student's t test).

\section{Results}

Summarized results from samples collected at the different pig slaughterhouses are shown in Table 1. The greatest TVC and EBC mean log values were found in samples from runoff pit and gut container collected during slaughter activities in slaughterhouse C. A TVC average up to $6 \log _{10} \mathrm{CFU} / \mathrm{cm}^{2}$ was detected in two slaughter plants (A and C), whereas a TVC average up to $5 \log _{10} \mathrm{CFU} / \mathrm{cm}^{2}$ was found in samples collected before slaughter activities from runoff pit and gut container in the slaughter plant $\mathrm{B}$. In addition, an EBC average up to $4.6 \log _{10}$ $\mathrm{CFU} / \mathrm{cm}^{2}$ was detected in samples collected from gut container before slaughter activities in the slaughterhouse A. The samples collected from runoff pit in the slaughterhouse $\mathrm{C}$ showed a high TVC and EBC mean log value both before and during slaughter activities. The logarithmic data for TVC and EBC counts from carcass samples are presented in Table 2 . Overall, mean log TVC ranged from 4.1 to 6.3 $\log _{10} \mathrm{CFU} / \mathrm{cm}^{2}$, whereas the EBC average ranged from 1.9 to $2.6 \log _{10} \mathrm{CFU} / \mathrm{cm}^{2}$. The Student t test showed statistically significant $(\mathrm{P}<0.05)$ differences in the bacterial counts between the carcasses tested in slaughterhouse A and those tested in the other two plants. Analysis of variance and the Sheffé post hoc tests showed that the carcasses produced by slaughterhouse A belong to a group that is completely separated from those of group B and C. Salmonella was recovered in two of the three slaughterhouses (A and B) during slaughter activities (Table 3). In details, Salmonella was recovered in samples collected from floor after the blee-

Table 1. Total viable count and Enterobacteriaceae.

\begin{tabular}{|c|c|c|c|c|c|c|c|}
\hline \multirow[t]{2}{*}{ Sampling place } & \multirow[t]{2}{*}{ Sampling time } & \multicolumn{2}{|c|}{ Slaughterhouse A } & \multicolumn{2}{|c|}{ Slaughterhouse B } & \multicolumn{2}{|c|}{ Slaughterhouse C } \\
\hline & & TVC & $\mathrm{EBC}$ & TVC & EBC & TVC & $\mathrm{EBC}$ \\
\hline \multirow[t]{2}{*}{ Floor after the bleeding stage } & During slaughter activities & $6.57 \pm 0.25$ & $4.06 \pm 0.48$ & $5.22 \pm 0.66$ & $3.29 \pm 0.93$ & $4.85 \pm 0.68$ & $2.30 \pm 0.61$ \\
\hline & Before slaughter activities & $4.46 \pm 0.23$ & $1.30 \pm 0.91$ & $4.42 \pm 0.57$ & $0.61 \pm 0.75$ & $4.30 \pm 0.86$ & $2.02 \pm 1.68$ \\
\hline \multirow[t]{2}{*}{ Runoff pit } & During slaughter activities & $7.16 \pm 1.41$ & $3.94 \pm 0.51$ & $4.91 \pm 0.60$ & $3.85 \pm 0.44$ & $7.86 \pm 0.68$ & $4.88 \pm 0.55$ \\
\hline & Before slaughter activities & $4.73 \pm 0.41$ & n.d. & $5.17 \pm 1.26$ & $1.59 \pm 1.93$ & $6.47 \pm 1.45$ & $2.49 \pm 0.08$ \\
\hline \multirow[t]{2}{*}{ Gut container } & During slaughter activities & $5.70 \pm 0.66$ & $3.75 \pm 1.09$ & $4.09 \pm 0.69$ & $3.04 \pm 0.97$ & $7.86 \pm 0.68$ & $1.58 \pm 1.38$ \\
\hline & Before slaughter activities & $6.0 \pm 0.26$ & $4.67 \pm 0.51$ & $3.07 \pm 0.78$ & n.d. & $4.26 \pm 1.09$ & $0.88 \pm 1.02$ \\
\hline
\end{tabular}

TVC, total viable count; EBC, Enterobacteriaceae; n.d., not detected [below detection limit $(<1$ colony forming unit/cm²)]. No statistically significant differences were found between sampling times for each point in each slaughterhouse. No statistically significant differences were found between slaughterhouses. 
ding stage and from gut container in the slaughterhouses A and B and in samples collected from runoff pit just in the slaughterhouse $B$. Additionally, Salmonella was isolated from 4/30 pre-chilled carcasses (13.3\%) in the slaughterhouse B. Among the selected batches, $2 / 6$ of them were positive for Salmonella (33.3\%) in the slaughterhouse B. No Salmonella was isolated from pooled samples of caecal contents. Four different Salmonella serotypes were detected in the positive samples (11): $S$. Rissen (45.4\%; 5/11), S. Derby (18.1\%; 2/11), $S$. Typhimurium $(9 \% ; 1 / 11)$ and the $S$. Typhimurium monophasic-variant (27.2\%; $3 / 11)$. $S$. Rissen and $S$. Derby were the serotypes among isolates recovered from carcasses while $S$. Rissen and the $S$. Typhimurium monophasic-variant were the serotypes among isolates recovered from environmental samples. Within serotype Rissen (5/11) two different PFGE restriction profiles could be distinguished. Among $S$. Typhimurium monophasicvariant isolates $(3 / 11)$, two PFGE patterns were identified, whereas the $S$. Derby strains (2/11) showed the same pulsotype. In the slaughterhouse B, $S$. Rissen isolates recovered from carcasses and from floor after bleeding stage showed the same PFGE profile.

\section{Discussion}

The aim of this survey was to obtain data on microbiological contamination of pig carcasses and slaughterhouse environment in three large-scale Italian slaughterhouses located in the Food Valley, where pigs are slaughtered for the production of Parma ham. Overall, the greatest TVC and EBC mean log values were detected both in environmental and carcass samples in the slaughterhouse A. The maintenance of good hygiene practices (GHP) and the effectiveness of the cleaning and disinfection plan depend on the qualification of the personnel and the structural features of the equipment. The results of environmental sampling did not show an association with the slaughterhouse plants. The microbiological analyses on the slaughterhouse environments showed that routine cleaning procedures are not able to remove microbiological contamination effectively: up to $6 \log _{10} \mathrm{CFU} / \mathrm{cm}^{2} \mathrm{TVC}$ (slaughterhouses $\mathrm{A}$ and $\mathrm{C}$ ) and $4.6 \log _{10}$ $\mathrm{CFU} / \mathrm{cm}^{2}$ EBC (slaughterhouse A) were detected in samples collected before the slaughter activities. In the slaughterhouse $\mathrm{C}$, samples collected from runoff pit showed a high TVC and EBC mean log values both before and during slaughter activities. Moreover, the hygienic conditions of animals have an important influence on the environmental hygiene. In the slaughterhouse A, the greatest contamination was detected in samples isolated from the floor after bleeding process, where animals fell on a horizontal platform after stunning. The results obtained in this survey could not be compared with published data: surveys differ in terms of sampling methods, sampling sites and evaluation modes (Hutchison et al., 2005; Zweifel et al., 2005, 2008; 0'Brien et al., 2007). In the present investigation, the mean TVC and the mean EBC ranged from 4.1 to 6.3 $\log _{10} \mathrm{CFU} / \mathrm{cm}^{2}$ and from 1.9 to $2.6 \log _{10} \mathrm{CFU} / \mathrm{cm}^{2}$ in the examined carcasses, respectively. In a recent study, Wheatley et al. (2014) examined the effects of eight processing stages during slaughtering line on contamination levels (by performing TVC and EBC). In total, 95 carcasses from an Irish pork slaughter plant were sampled by swabbing $100 \mathrm{~cm}^{2}$ of surface at three sites (belly, ham and jowl). In details, TVC ranged approximately from 1.7 to $6.3 \log _{10}$ $\mathrm{CFU} / \mathrm{cm}^{2}$ during sampling. In particular, following the final inspection and before chilling the TVC level was approximately $3.0 \log _{10}$ $\mathrm{CFU} / \mathrm{cm}^{2}$. However, after the final inspection and chilling the EBC level was relatively higher (2.0-2.8 $\log _{10} \mathrm{CFU} / \mathrm{cm}^{2}$ ) compared with previous stages. In our survey, the greatest TVC $\left(\begin{array}{lllll}6.3 & \log _{10} & \mathrm{CFU} / \mathrm{cm}^{2}\end{array}\right)$ and EBC (2.6 $\log _{10}$ $\mathrm{CFU} / \mathrm{cm}^{2}$ ) mean $\log$ value on carcasses was found in slaughterhouse $\mathrm{A}$, where an average up to $6 \log _{10} \mathrm{CFU} / \mathrm{cm}^{2} \mathrm{TVC}$ and $4.6 \log _{10} \mathrm{CFU} / \mathrm{cm}^{2}$ EBC was found in samples collected before slaughter activities. The Student t test showed statistically significant $(\mathrm{P}<0.05)$ differences between the carcasses tested in slaughterhouse A and those tested in the other two slaughter plants (B-C). The Sheffé post hoc test, in fact, showed that the carcasses produced by slaughterhouse A lay in a group which is completely separated from those of group B and C. Based on this results, carcass contamination seems to be influenced by the slaughterhouse plant and this may be a result of differences in line speed. However, it may also reflect other differences, such as animal selection, the good slaughter practices employed, the competence of the slaughter staff etc. A variation in Salmonella contamination of the environment and the carcasses among the different pig slaughter plants (A-B-C) was observed. These results may depend on the inhomogeneous application of GHP by the operators. An investigation performed in five abattoirs reported that Salmonella was isolated from 13 of 41 environmental samples (31.7\%) at the end of the sampling day and before cleaning (Piras et al., 2011). According to Piras et al. (2011), the sampling results depend on the slaughterhouse, the sampling day and the origin and number of infected pigs delivered during the sample period. Salmonella was recovered in two (A and B) of the three slaughterhouses. In particular, Salmonella was recovered during two different sampling visits in the slaughterhouse A from floor after the bleeding stage and gut container, and during three different sampling visits in the slaughterhouse B from floor after the bleeding stage, runoff pit an gut container. Additionally, Salmonella was isolated from environmental and carcass samples during the same sampling day in the slaughterhouse B. No Salmonella was isolated in the carcass

Table 2. Total viable count and Enterobacteriaceae on pig carcasses at slaughterhouses A, B, C.

\begin{tabular}{|c|c|c|c|}
\hline Slaughterhouses & Number of carcasses analysed & TVC $\left(\log _{10} \mathrm{CFU} / \mathrm{cm}^{2}\right)$ & EBC $\left(\log _{10} \mathrm{CFU} / \mathrm{cm}^{2}\right)$ \\
\hline A & 30 & $6.3 \pm 0.201^{\mathrm{a}}$ & $2.6 \pm 0.333^{\mathrm{a}}$ \\
\hline B & 30 & $4.1 \pm 0.212^{b}$ & $2.0 \pm 0.223^{b}$ \\
\hline $\mathrm{C}$ & 30 & $4.6 \pm 0.724^{b}$ & $1.9 \pm 0.953^{\mathrm{b}}$ \\
\hline
\end{tabular}

TVC, total viable count; EBC, Enterobacteriaceae; CFU, colony forming unit. Data are expressed as mean $\log _{10}$ CFU \pm standard deviation. ${ }^{\text {a,b }}$ Different superscript letters denote statistically significant differences (Student's t test; $\mathrm{P}<0.05$ ) in count (TVC and $\mathrm{EBC}$ ) on carcasses from the three slaughter plants.

Table 3. Prevalence of Salmonella from pig slaughterhouses A, B and C.

\begin{tabular}{lccc} 
& Slaughterhouse A & Slaughterhouse B \\
Carcass sponge & $0 / 30(0)$ & $4 / 30(13.3 \%)$ & Slaughterhouse C \\
Caecal content & $0 / 6(0)$ & $0 / 6(0)$ & $0 / 30(0)$ \\
\hline Environmental samples & $2 / 36(5.5 \%)^{\circ}$ & $5 / 36(13.8 \%)^{\circ}$ & $0 / 36(0)$ \\
\hline
\end{tabular}

${ }^{\circ}$ All Salmonella positive samples were collected during slaughter activities. 
and/or environmental samples in the slaughterhouse $\mathrm{C}$. This result may be explained by the fact that, in the slaughterhouse $\mathrm{C}$ there is a break (about 15 minutes) after 4 hours of activity: before the renewal of activity, a cleaning step with water is performed. This seems to be very efficient to control the environment contamination by Salmonella. Moreover, in our investigation the contamination of Salmonella (4.44\%) resulted lower than that $(37 \%)$ previously reported by some authors. On the other hand, our findings are in accordance with other studies (Piras et al., 2011; Arguello et al., 2013; Milios et al., 2014). However, comparisons among different studies on Salmonella contamination of carcasses should be considered carefully because prevalence estimates are affected by the isolation procedures and particularly by the sampling strategy: the location and the area of the carcass surface sampled or the point of the slaughtering process at which the swabs are taken. In the present survey, no Salmonella could be isolated from pooled caecal samples. Carcass contamination can be affected by the pig status and also by several internal factors of the slaughtering process (Arguello et al., 2014). In the present survey, different Salmonella serotypes were detected in the positive samples ( $S$. Rissen, $S$. Derby, $S$. Typhimurium and the $S$. Typhimurium monophasic-variant). These serotypes are the most frequently isolated from pig and pork in many countries (EFSA, 2008a, 2008b, 2009). Furthermore, this study showed that the same Salmonella serotypes could be identified in samples collected from both the carcasses and the environment in the slaughterhouse B. As for the PFGE analysis, a diversity of strains has been identified since two different PFGE profiles were isolated from slaughterhouse $\mathrm{A}$ and five from slaughterhouse B. The diversity among the PFGE profiles may be linked to the high slaughter capacity (approximately 350 pigs/hour in the slaughterhouses $B$ and $C$ and 450 pigs/hour in the slaughterhouse A). However, it was not possible to isolate the same pulsotype within the same slaughterhouse in different sampling days. In the slaughterhouse B, $S$. Rissen isolates recovered from carcasses and from floor after bleeding showed the same PFGE profile. This finding could suggest that carcass contamination may be occurred through slaughterhouse environment. After stunning, in fact, pigs fell on a horizontal conveyor belt where they were bled whilst the animals were prone on the platform (floor after bleeding stage). A regular cleaning and disinfecting during slaughter process of the floor after bleeding stage could avoid, or at least decrease, cross-contamination of carcass. Our survey highlighted that the GHP employed in these high processing speed slaughterhouses (up to 450 pigs/hour) should be implemented by the cleaning staff.

\section{Conclusions}

In order to protect human health against Salmonella infections transmissible between animals and humans, the Regulation (EC) n. 2160/2003 (European Commission, 2003) made national control plans for Salmonella serovars in poultry and pigs mandatory between Member States. The findings in this survey may suggest that carcass contamination is influenced by slaughterhouse and this could be a result of differences in line speed. However, they could also reflect other differences such as animal selection, the good slaughter practices employed, the competence of the slaughter staff, etc. As for as the slaughter environment is concerned, areas with different degrees of contamination were detected. The results of environmental sampling do not show an association with slaughterhouse. This survey indicates that the routine slaughter and hygiene practices employed in the large-scale Italian pig slaughterhouses with high processing speed should be implemented. Additionally, in order to prevent carcass contamination the slaughterhouse managers should evaluate the effectiveness of the sanitation standard operating procedures (SSOP) and revise these procedures to keep them effective and current with respect to the slaughter line speed (up to 450 carcasses/hour). An improved cleaning protocol, including the use of disinfectants, and a proper instruction of personnel are the main tools to decrease Salmonella contamination at slaughtering stage. Employee training is equally important as the SSOP procedures. The competence of the slaughter and cleaning staff is pivotal to minimize carcass and environment contamination in slaughterhouses. The slaughterhouse managers should bear in mind the weak points in the hygienic design, including places where organic residues may accumulate and microbes may persist in the environment as biofilm.

\section{References}

Arguello H, Alvarez-Ordonez A, Carvajal A, Rubio P, Prieto M, 2013. Role of slaughtering in Salmonella spreading and control in pork production. J Food Protect 76:899911.

Arguello H, Carvajal A, Alvarez-0rdonez A, Jaramillo-Torres HA, Rubio P, 2014. Effect of logistic slaughter on Salmonella contamination on pig carcasses. Food Res Int 55:77-82.

Duggan S J, Mannion C, Prendergast DM, Leonard N, Fanning S, Gonzales-Barron U, Egan J, Butler F, Duffy G, 2010. Tracking the Salmonella status of pigs and pork from lairage through the slaughter process in the Republic of Ireland. J Food Protect 73:2148-60.

European Commission, 2003. Regulation (EC) No 2160/2003 of the European Parliament and of the Council of 17 November 2003 on the control of Salmonella and other specified food-borne zoonotic agents. In: Official Journal, L 325, pp. 1-15.

European Commission, 2005. Regulation (EC) No. 2073/2005 of 15 November 2005 on microbiological criteria for foodstuffs. In: Official Journal, L 338, pp. 1-26.

European Commission, 2014. Regulation (EU) No. 217/2014 of 7 March 2014 amending Regulation (EC) No 2073/2005 as regards Salmonella in pig carcasses. In: Official Journal, L 69, pp. 93-94.

Edwards PR, 1962. Serologic examination of Salmonella cultures for epidemiologic purposes. National Communicable Disease Center, Atlanta, GA, USA.

EFSA, 2008a. Report of the task force on zoonoses data collection on the analysis of the baseline survey on the prevalence of Salmonella in slaughter pigs, in the EU, 2006-2007, Part A: Salmonella prevalence estimates. EFSA J 135:1-111.

EFSA, 2008b. Report of the task force on zoonoses data collection on the analysis of the baseline survey on the prevalence of Salmonella in slaughter pigs, Part B. EFSA J 206:1-111.

EFSA, 2009. Analysis of the baseline survey on the prevalence of Salmonella in holdings with breeding pigs in the EU, 2008. Part A: Salmonella prevalence estimates. EFSA J 7:1377.

EFSA, 2012. The European Union summary report on trends and sources of zoonoses, zoonotic agents and food-borne outbreaks in 2010. European Food Safety Authority, Parma, Italy.

Gomes-Neves E, Antunes P, Tavares A, Themudo P, Cardoso MF, Gartner F, Costa JM, Peixe L, 2012. Salmonella cross-contamination in swine abattoirs in Portugal: carcasses, meat and meat handlers. Int $\mathrm{J}$ Food Microbiol 157:82-7.

Grimont PAD, Weill FX, 2007. Antigenic formulae of the Salmonella serovars, 9th ed. WHO Collaborating Center for Reference and Research on Salmonella, Institut Pasteur, Paris, France.

Hutchison ML, Walters LD, Avery SM, Reid CA, Wilson D, Howell M, Johnston AM, Buncic S, 2005. A comparison of wet-dry swabbing and excision sampling methods for microbiological testing of bovine, porcine, and ovine carcasses at red meat slaughterhouses. J Food Protect 68:2155-62.

Milios K, Drosinos EH, Zoiopoulos PE, 2014. Food safety management system validation and verification in meat industry: car- 
cass sampling methods for microbiological hygiene criteria. A review. Food Control 43:74-81.

Morris GK, Steele CD, Wells JG, 1972. Evaluation of plastic multi-well plates for serological screening of Salmonella cultures with Spicer-Edwards pooled antisera. Appl Microbiol 24:846-8.

0'Brien SB, Lenahan M, Sweeney T, Sheridan JJ, 2007. Assessing the hygiene of pig carcasses using whole-body carcass swabs compared with the four-site method in EC Decision 471. J Food Protect 70:432-9.

Piras F, Brown DJ, Meloni D, Mureddu A, Mazzette R, 2011. Investigation of Salmonella enterica in Sardinian slaughter pigs: prevalence, serotype and genotype characterization. Int J Food Microbiol 151:201-9.
PULSENET, 2010. One day (24-28h) Standardized Laboratory Protocol for Molecular Subtyping of Escherichia coli 0157:H7, Salmonella serotypes, Shigella sonnei and Shigella flexneri by Pulsed Field Gel Electrophoresis (PFGE). Pulsenet International 2010:1-16.

Spicer CC, 1956. A quick method of identifying Salmonella $\mathrm{H}$ antigens. J Clin Pathol 9:378-9.

Valero A, Hernandez M, De Cesare A, Manfreda G, Garcia-Gimeno RM, Gonzalez-Garcia P, Rodriguez-Lazaro D, 2014. Probabilistic approach for determining Salmonella spp. and L. monocytogenes concentration in pork meat from presence/absence microbiological data. Int $\mathrm{J}$ Food Microbiol 184:60-3.

Wheatley P, Giotis ES, McKevitt AI, 2014.
Effects of slaughtering operations on carcass contamination in an Irish pork production plant. Irl Vet J 67:1.

Wilhelm B, Rajic A, Greig JD, Waddell L, Harris J, 2011. The effect of hazard analysis critical control point programs on microbial contamination of carcasses in abattoirs: a systematic review of published data. Foodborne Pathog Dis 8:949-60.

Zweifel C, Baltzer D, Stephan R, 2005. Microbiological contamination of cattle and pig carcasses at five abattoirs determined by swab sampling in accordance with EU Decision 2001/471/EC. Meat Sci 69:559-66.

Zweifel C, Fischer R, Stephan R, 2008. Microbiological contamination of pig and cattle carcasses in different small-scale Swiss abattoirs. Meat Sci 78:225-31. 